\title{
Multi-Objective Restricted Solid Transportation Problem in Intuitionistic Fuzzy Environment with Emission Cost
}

\author{
P. Vijayalakshmi, J. Merline Vinotha
}

\begin{abstract}
Transportation plays key role in logistic and supply chain management for decreasing cost and enhances service. The transport sector contributes $23 \%$ of the total $\mathrm{CO} 2$ emissions in the world according to the latest estimates of the International Energy Agency (IEA).There is a direct link between weight of the quantity transported and co2 emission for the freight transport. This paper presents multi objective restricted solid transportation problem in intuitionistic fuzzy ambiance with emission cost which is based on weight of the quantity transported and vehicle cost under some restriction on transported amount. An extra constraint on the total budget at each destination is imposed. Transportation models are formulated under crisp and fuzzy environments and fuzzy models are converted into crisp using average method. The total time and emission cost based on weight of the quantity transported for restricted and unrestricted models are compared. The optimal solution is obtained by using weighted sum method and Lingo 13.0 Software. Mathematical example is given to validate the proposed model.
\end{abstract}

Keywords: Multi-objective, Solid Transportation Problem, Budget constraint, Atanassov's intuitionist fuzzy Number, emission cost.

\section{INTRODUCTION}

The conventional transportation problems (TP) are aspired to resolve the most advantageous way to reduce the transportation cost. Hitchcock (1941) initiated the term transportation problem, by modeling it like a conventional optimization problem with two dimensional characters that is supply and demand. Apart from supply and demand in real world system, frequently another dimension related to the mode of transportation, types of commodities etc. Under such situations, a TP is extended to a solid transportation problem (STP). Schell (1955)[17] introduced solid transportation problem and Haley (1963) brought the further developments in $\operatorname{STP}[12,13]$

In the current framework single objective transportation problems are not succeed to meet up the managerial decision creation requirements which demands the multi objective transportation problems. Numerous researchers suggested the methodologies to solve transportation problem with multi objectives.[1,2]

Furthermore the associated parameters for such problems may be vague due to incompleteness or lack of proof, statistical analysis, etc. As a result, in such cases, the fuzzy number can be used to resolve ambiguity in data. Many researchers have illustrated the significance of Atanassov's intuitionistic fuzzy sets / numbers and its application in optimization Problem ([4], [8], [9]). Hussain et al. [16] and Gani et al.[3,5]discussed the method of solving Atanassov's intuitionistic fuzzy transportation problem.

The carbon dioxide emissions from the freight transport are the most important reason for global warming. Several green logistics works attempt to reduce the carbon emissions which are related to the load on vehicles. The best way to reduce the co2 emission is increasing the load. The major determinants of the load limitations are vehicle capacity and vehicle cost.[7]

Hence evolving the multi objective solid transportation with the restriction on the amount of quantity transported and the emission cost based on the weight of the quantity transported would be helpful to the society because of the reduction of $\operatorname{co} 2$ emission is directly related to the load. In this model unit transportation costs, supply, demand, capacity of the conveyances, budget at each destination and emission cost based on weight of the quantity transported are triangular Atanassov's intuitionistic fuzzy number.

The rest of the study is categorized as follows: Preliminaries are stated in section 2.The procedure to reduce multi objective transportation problem into single objective is discussed in section 3.Assumptions and notations are given in section 4.Mathematical formulation and the solution methodology are presented in section 5.Numerical example is given in section 6. Results and discussions are presented in section 7\&8. Sensitivity analysis is given in section 9 . Conclusion is given in section 10 .

\section{PRELIMINARIES:}

\section{Definition:2.1 :Atanassov's Intuitionistic Fuzzy Set} (A-IFS)

Let $\mathrm{E}$ be a given set and $e \mathrm{~A} \subset \mathrm{E}$ be a set. An $\mathrm{A}-\mathrm{IFS} e \mathrm{~A}^{*}$ in $\mathrm{E}$ is given by

$$
e \mathrm{~A}^{*}=\left\{\left\langle e x, \mu_{e \mathrm{~A}}(e x), \mathrm{n}_{e \mathrm{~A}}(e x)\right\rangle ; e x \in \mathrm{E}\right\} \text { where }
$$
TN, India.

Revised Version Manuscript Received on July 10, 2019.

P. Vijayalakshmi, Hindusthan Institute of Technology, Coimbatore, India

J. Merline Vinotha, Holy Cross College (Autonomous), Trichy,TN, 


\section{MULTI-OBJECTIVE MULTI TIME SPAN FRACTIONAL CAPACITATED TRANSPORTATION PROBLEM IN THE TWO ECHELON SUPPLY CHAIN WITH MULTIFORM ITEMS AND MIXED CONSTRAINTS}

$\mu e_{\mathrm{A}}: \mathrm{E} \rightarrow[0,1]$ and $\mathrm{n}_{e \mathrm{~A}}: \mathrm{E} \rightarrow[0,1]$ define the degree of membership and the degree of non-membership of the element $e x \in \mathrm{E}$ to $e \mathrm{~A} \subset \mathrm{E}$ satisfy the condition $\mathrm{O}$

$$
\leq \mu_{e}(e x)+\mathrm{n}_{e \mathrm{~A}}(e x) \leq 1 .
$$

Definition:2.2 :Atanassov's Intuitionistic Fuzzy Number $(A-I F N)$

An Atanassov'sintuitionisticfuzzy Subset $e \tilde{\mathrm{A}}^{\prime}=\left\{\left\langle e x, \mu_{e \tilde{\mathrm{A}}^{\prime}}, \mathrm{n}_{e \tilde{\mathrm{A}}^{\prime}}\right\rangle\right\} \quad$ of the real line $\hat{\mathrm{A}}$ is called A-IFN, if the followings holds.

(i) there exist $\mathrm{m} \in \hat{\mathrm{A}}, \mu_{e \tilde{\AA}^{\prime}}(\mathrm{m})=1, \mathrm{n}_{e \tilde{\mathrm{A}}^{\prime}}(\mathrm{m})=0$.

(ii) $\mu_{e \tilde{\mathrm{A}}^{\prime}}$ is continuous mapping from $\hat{\mathrm{A}}$ to the closed interval $[0,1]$ and $e x \in \hat{\mathrm{A}}$, the relation $0 \leq \mu_{e \tilde{\mathrm{A}}^{\prime}}(e x)+\mathrm{n}_{e \tilde{\mathrm{A}}^{\prime}}(e x) \leq 1$ holds.

The membership function and non-membership function of is of the following form.

$$
\mu_{e \tilde{A}^{\prime}}(e x)=\left\{\begin{array}{l}
\mathrm{f}_{1}(e x), \quad \mathrm{m}-\alpha \leq e x \leq \mathrm{m} ; \\
1, \quad \text { ex }=\mathrm{m} ; \\
\mathrm{h}_{1}(e x), \mathrm{m} \leq \text { ex } \leq \mathrm{m}+\beta ; \\
0, \quad \text { Otherwise. }
\end{array}\right.
$$

Non-membership Function :

$$
\mathrm{n}_{e \tilde{A}^{\prime}}(e x)= \begin{cases}\mathrm{f}_{2}(e x), & \mathrm{m}-\alpha^{\prime} \leq e x \leq \mathrm{m}, \\ & 0 \leq\left(\mathrm{f}_{1}(e x)+\mathrm{f}_{2}(e x)\right) \leq 1 ; \\ 0, & e x=\mathrm{m} ; \\ \mathrm{h}_{2}(e x), & \mathrm{m} \leq e x \leq \mathrm{m}+\beta^{\prime}, \\ & 0 \leq\left(\mathrm{h}_{1}(e x)+\mathrm{h}_{2}(e x)\right) \leq 1 ; \\ 1, & \text { Otherwise. }\end{cases}
$$

Here $\mathrm{f}_{1}(\mathrm{e} x)$ and $\mathrm{h}_{1}(\mathrm{e} x)$ are rigorously increasing and decreasing function in $[\mathrm{m}-\alpha, \mathrm{m}]$ and $[\mathrm{m}, \mathrm{m}+\beta]$ correspondingly and $\mathrm{f}_{2}(\mathrm{e} x)$ and $\mathrm{h}_{2}(\mathrm{e} x)$ are severely diminishing and increasing function in $\left[\mathrm{m}-\alpha^{\prime}, \mathrm{m}\right]$ and $[\mathrm{m}, \mathrm{m}+$ $\beta^{\prime}$ ] respectively, where $m$ is the mean value of $e \tilde{\mathrm{A}}^{\prime} . \alpha$ and $\beta$ are called the left and accurate spreads of membership function $\mu_{e \tilde{\mathrm{A}}^{\prime}}(e x)$ respectively. $\alpha^{\prime}$ and $\beta^{\prime}$ are called the left and right spreads of non-membership function $\mathrm{n}_{e \tilde{\mathrm{A}}^{\prime}}(e x)$ respectively.

Definition 2.3: Triangular Atanassov's Intuitionistic Fuzzy Number (TrIFN) :

Let $e \mathrm{a}_{1}^{\prime} \leq e \mathrm{a}_{1} \leq e \mathrm{a}_{2} \leq e \mathrm{a}_{3} \leq e \mathrm{a}_{3}^{\prime}$. ATrIFN $e \tilde{\mathrm{A}}^{\prime}$ in $\hat{\mathrm{A}}$ written as $\left(e \mathrm{a}_{1}, \mathrm{ea}_{2}, \mathrm{ea}_{3}\right)\left(e \mathrm{a}_{1}^{\prime}, e \mathrm{a}_{2}, e \mathrm{a}_{3}^{\prime}\right)$ has membership function (c.f. Fig.1)

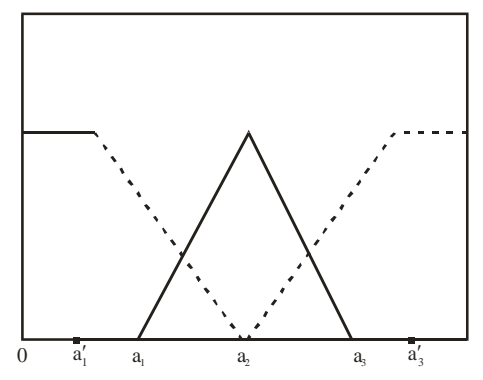

Fig.1. Membership and Non-membership Function of TrIFN

$$
\mu_{e \tilde{A}^{\prime}}(e x)= \begin{cases}\frac{e x-e \mathrm{a}_{1}}{e \mathrm{a}_{2}-e \mathrm{a}_{1}}, & e \mathrm{a}_{1} \leq e x \leq e \mathrm{a}_{2} \\ 1, & e x=e \mathrm{a}_{2} \\ \frac{e \mathrm{a}_{3}-e x}{e \mathrm{a}_{3}-e \mathrm{a}_{2}}, & e \mathrm{a}_{2} \leq e x \leq e \mathrm{a}_{3} \\ 0, & \text { Otherwise. }\end{cases}
$$

Non-membership function:

$$
\mathrm{n}_{e \tilde{\mathrm{A}}^{\prime}}(e x)= \begin{cases}\frac{e \mathrm{a}_{2}-e x}{e \mathrm{a}_{2}-e \mathrm{a}_{1}^{\prime}}, & e \mathrm{a}_{1}^{\prime} \leq e x \leq e \mathrm{a}_{2} \\ 0, & e x=e \mathrm{a}_{2} \\ \frac{e x-e \mathrm{a}_{2}}{e \mathrm{a}_{3}^{\prime}-e \mathrm{a}_{2}}, & e \mathrm{a}_{2} \leq e x \leq e \mathrm{a}_{3}^{\prime} \\ 1, & \text { Otherwise. }\end{cases}
$$

Definition 2.4:

A positive TrIFN is denoted as $e \tilde{\mathrm{A}}^{\prime}=\left(e \mathrm{a}_{1}, e \mathrm{a}_{2}\right.$, $\left.e \mathrm{a}_{3}\right)\left(e \mathrm{a}_{1}^{\prime}, e \mathrm{a}_{2}, e \mathrm{a}_{3}^{\prime}\right)$ where $e \mathrm{a}_{1}^{\prime}>0$. Then we define $e \tilde{\mathrm{A}}^{\prime}$ $=\frac{1}{8}\left[\left(e \mathrm{a}_{1}+2 e \mathrm{a}_{2}+e \mathrm{a}_{3}\right)+\left(e \mathrm{a}_{1}^{\prime}+2 e \mathrm{a}_{2}+e \mathrm{a}_{3}^{\prime}\right)\right]$

an accuracy function of , to defuzzify the given number.

Definition 2.5:

Let $e \tilde{\mathrm{A}}^{\prime}=\left(e \mathrm{a}_{1}, e \mathrm{a}_{2}, e \mathrm{a}_{3}\right)\left(e \mathrm{a}_{1}^{\prime}, e \mathrm{a}_{2}, e \mathrm{a}_{3}^{\prime}\right)$ and $e \tilde{\mathrm{B}}^{\prime}=$ $\left(e \mathrm{~b}_{1}, e \mathrm{~b}_{2}, e \mathrm{~b}_{3}\right)\left(e \mathrm{~b}_{1}^{\prime}, e \mathrm{~b}_{2}, e \mathrm{~b}_{3}^{\prime}\right)$ be the two TrIFN then

(i) $e \tilde{\mathrm{A}}^{\prime} \oplus e \tilde{\mathrm{B}}^{\prime}=$

$\left(e \mathrm{a}_{1}+e \mathrm{~b}_{1}, e \mathrm{a}_{2}+e \mathrm{~b}_{2}, e \mathrm{a}_{3}+e \mathrm{~b}_{3}\right)\left(e \mathrm{a}_{1}^{\prime}+e \mathrm{~b}_{1}^{\prime}, e \mathrm{a}_{2}+e \mathrm{~b}_{2}, e \mathrm{a}_{3}^{\prime}+e \mathrm{~b}_{3}^{\prime}\right)$

(ii) $\mathrm{Ke} e \tilde{\mathrm{A}}^{\prime}=\left(\mathrm{Kea}_{1}, \mathrm{Kea}_{2}, \mathrm{Kea}_{3}\right)\left(\mathrm{Kea}_{1}^{\prime}, \mathrm{Kea}_{2}, \mathrm{Kea}_{3}^{\prime}\right)$ if $\mathrm{K} \geq 0$.

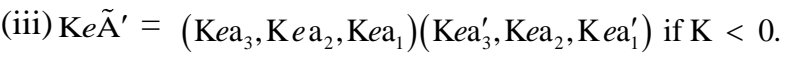

(iv) $e \tilde{\mathrm{A}}^{\prime} \quad \tilde{\mathrm{B}}^{\prime}=$

$\left(e \mathrm{a}_{1}-e \mathrm{~b}_{3}, e \mathrm{a}_{2}-e \mathrm{~b}_{2}, e \mathrm{a}_{3}-e \mathrm{~b}_{1}\right)\left(e \mathrm{a}_{1}^{\prime}-e \mathrm{~b}_{3}^{\prime}, e \mathrm{a}_{2}-e \mathrm{~b}_{2}, e \mathrm{a}_{3}^{\prime}-e \mathrm{~b}_{1}^{\prime}\right)$

(v) $e \tilde{\mathrm{A}}^{\prime} \otimes e \tilde{\mathrm{B}}^{\prime}=$

$\left(e \mathrm{a}_{1} e \mathrm{~b}_{1}, e \mathrm{a}_{2} e \mathrm{~b}_{2}, e \mathrm{a}_{3} e \mathrm{~b}_{3}\right)\left(e \mathrm{a}_{1}^{\prime} e \mathrm{~b}_{1}^{\prime}, e \mathrm{a}_{2} e \mathrm{~b}_{2}, e \mathrm{a}_{3}^{\prime} e \mathrm{~b}_{3}^{\prime}\right)$

\section{METHODOLOGY TO REDUCE THE} MULTI-OBJECTIVE TRANSPORTATION MODELS INTO SINGLE

Objective transportation models (Weighted Sum Method) 
The weighted sum method is used to change multi objective transportation models into single objective transportation models. A compound objective function $\mathrm{Z}$ can be constructing by summing the weighted standardized objectives and the MOSTP is then transformed to a single-objective optimization problem as follows:

$$
\text { Minimize } \mathrm{Z}=\sum_{\mathrm{r}=1}^{\mathrm{L}} \mathrm{w}_{\mathrm{r}} e \mathrm{f}_{\mathrm{r}}, \mathrm{w}_{\mathrm{r}} \in[0,1]
$$

Here,wr is the weight of the rth objective function. Since the minimum of the above problem does not change if all the

weights are multiplied by a constant, so to choose weights such that sum of their weight is equal to 1 (i.e.,) $\sum_{\mathrm{r}=1}^{\mathrm{L}} \mathrm{w}_{\mathrm{r}}=1$. In MOSTP the objective functions are conflict with each other. Here the non-dominated optimality approach is imported because an complete optimal solution does not always exist."

\section{ASSUMPTIONS AND NOTATIONS}

The following notation and assumption are used throughout the model.

(i) $e \mathrm{C}_{\mathrm{IJK}}, e \tilde{\mathrm{C}}_{\mathrm{IJK}}$ : crisp, fuzzy unit transportation cost to transport the commodity from $\mathrm{I}^{\text {th }}$ origin to $\mathrm{J}^{\text {th }}$ destination by $\mathrm{K}^{\text {th }}$ conveyances respectively.

(ii) $e \mathrm{t}_{\mathrm{IJK}}, e \tilde{\mathrm{t}}_{\mathrm{IJK}}:$ crisp, fuzzy transportation time to $\mathrm{K}^{\text {th }}$ conveyances respectively.

(iii) $e \mathrm{a}_{\mathrm{I}}, e \tilde{\mathrm{a}}_{\mathrm{I}}$ : crisp, fuzzy amount of homogeneous product available at $\mathrm{I}^{\text {th }}$ origin respectively.

(iv) $e \mathrm{~b}_{\mathrm{J}}, e \tilde{\mathrm{b}}_{\mathrm{J}}$ : crisp, fuzzy amount of homogeneous product available at $\mathrm{J}^{\text {th }}$ destination respectively.

(v) $\mathrm{e}_{\text {IJK }}, \tilde{\mathrm{e}}_{\text {IJK }}$ : crisp, fuzzy emission cost based on weight to transport the commodity from $\mathrm{I}^{\text {th }}$ origin to $\mathrm{J}^{\text {th }}$ destination by $\mathrm{K}^{\text {th }}$ conveyances respectively.

(vi) $e \mathrm{e}_{\mathrm{K}}, e \tilde{\mathrm{e}}_{\mathrm{K}}$ : crisp, fuzzy amount of product which can be carried by the $\mathrm{K}^{\text {th }}$ conveyances respectively.

(vii) $e \mathrm{~B}_{\mathrm{J}}, e \tilde{\mathrm{B}}_{\mathrm{J}}$ : crisp, fuzzy available budget at $\mathrm{J}^{\text {th }}$ destination respectively.

(viii) $^{e x_{\text {गK }}}$ : Unknown quantity which is to be transport the commodity from $\mathrm{I}^{\text {th }}$ origin to $\mathrm{J}^{\text {th }}$ destination by $\mathrm{K}^{\text {th }}$ conveyance (decision variable).

(ix) If the unknown quantity which is to be transported from $\mathrm{I}^{\text {th }}$ source to $\mathrm{J}^{\text {th }}$ destination by $\mathrm{K}^{\text {th }}$ conveyance is $e x_{\text {एК }}>0$ then for the convenience of modeling we define

$$
\begin{aligned}
& e y_{\mathrm{IK}} \text { as follows : } \\
& e y_{\mathrm{IKK}}= \begin{cases}1 & \text { for } e x_{\mathrm{IJK}}>0 \\
0 & \text { Otherwise }\end{cases}
\end{aligned}
$$

(x) If in a specific destination the insignificant sum of quantity ( $\mathrm{p}$, say) is desired then the decision maker (DM) cannot deliver commodities in the particular destination. This means, if $e x_{\mathrm{\nu K}} \geq p$, a desired real number, and then we think transport the commodity from $\mathrm{I}^{\text {th }}$ origin to $\mathrm{J}^{\text {th }}$ destination by

the constraint for this direction as a part of the transportation. Consequently for the expediency of modeling, the following notation is introduced :

$$
e z_{\mathrm{IJK}}=\left\{\begin{array}{l}
1 \quad \text { for } e x_{\mathrm{IJK}} \geq \mathrm{p} \\
0 \quad \text { Otherwise }
\end{array},\right.
$$

(xi) It is important to note that the emission cost depends on the quantity transported. For less than 10 ton load the emission level is $255 \mathrm{~g} / 1 \mathrm{lr}$ diesel and for greater than 10 ton load the emission level is $180 \mathrm{~g} / 1 \mathrm{lr}$ diesel.

\section{Mathematical Formulation:}

\subsection{Model - I}

Multi-Objective Solid Transportation Problem (MOSTP) with emissioncost, budget constraint and vehicle cost in crisp environment:

In this model emission cost based on weight and budget of customers plays a vital role in transportation problem. Here we formulate MOSTP with M-origin, N-destination and $\mathrm{K}$-conveyances as follows :

$$
\begin{aligned}
& \text { Min } \mathrm{f}_{1}=\sum_{\mathrm{I}=1}^{\mathrm{M}} \sum_{\mathrm{J}=1}^{\mathrm{N}} \sum_{\mathrm{K}=1}^{\mathrm{L}} e \mathrm{C}_{\mathrm{IJK}} e x_{\mathrm{IJK}}+\sum_{\mathrm{I}=1}^{\mathrm{M}} \sum_{\mathrm{J}=1}^{\mathrm{N}} \sum_{\mathrm{K}=1}^{\mathrm{L}} \mathrm{V}\left(e x_{\mathrm{IJK}}\right) \text {, } \\
& \operatorname{Min} \mathrm{f}_{2}=\sum_{\mathrm{I}=1}^{\mathrm{M}} \sum_{\mathrm{J}=1}^{\mathrm{N}} \sum_{\mathrm{K}=1}^{\mathrm{L}} e \mathrm{t}_{\mathrm{IJK}} e \mathrm{y}_{\mathrm{IJK}}, \operatorname{Min}_{3}=\sum_{\mathrm{I}=1}^{\mathrm{M}} \sum_{\mathrm{J}=1}^{\mathrm{N}} \sum_{\mathrm{K}=1}^{\mathrm{L}} \mathrm{e}_{\mathrm{IJK}} e \mathrm{y}_{\mathrm{IJK}}
\end{aligned}
$$

Subject to constraints,

$$
\begin{aligned}
& \sum_{\mathrm{J}=1}^{\mathrm{N}} \sum_{\mathrm{K}=1}^{\mathrm{L}} e x_{\mathrm{IJK}} \leq e \mathrm{a}_{\mathrm{I}} \\
& \sum_{\mathrm{I}=1 \mathrm{~K}=1}^{\mathrm{M}} \sum_{\mathrm{L}}^{\mathrm{L}} e x_{\mathrm{IJK}} \geq e \mathrm{~b}_{\mathrm{J}} \\
& \sum_{\mathrm{I}=1}^{\mathrm{M}} \sum_{\mathrm{J}=1}^{\mathrm{N}} e x_{\mathrm{IJK}} \leq e \mathrm{e}_{\mathrm{K}} \\
& \sum_{\mathrm{I}=1 \mathrm{~K}=1}^{\mathrm{L}} \sum_{e x_{\mathrm{JK}} \geq 0}^{\mathrm{L}} e \mathrm{c}_{\mathrm{IJK}} e x_{\mathrm{IJK}} \leq e \mathrm{~B}_{\mathrm{J}} \\
&
\end{aligned}
$$
cost for the quantity ${ }^{e x}$ एк from $\mathrm{I}^{\text {th }}$ source OI, to $\mathrm{J}^{\text {th }}$ destination $\mathrm{DJ}$ via $\mathrm{K}^{\mathrm{th}}$ conveyances is defined as

$$
\mathrm{V}\left(e x_{\mathrm{IJK}}\right)=\left\{\begin{array}{ll}
\mathrm{m} \cdot \mathrm{v}, & \text { if } \mathrm{m} \cdot \mathrm{v}_{\mathrm{c}}=e x_{\mathrm{IJK}} \\
(\mathrm{m}+1) \mathrm{v}, & \text { otherwise }
\end{array}, \mathrm{m}=\left[e x_{\mathrm{IJK}} \mid \mathrm{v}_{\mathrm{c}}\right],\right.
$$

$\mathrm{V}_{\mathrm{c}}=$ Vehicle capacity and $\mathrm{V}=$ Vehicle Cost."

\subsection{Model - II}

Restricted Multi-Objective Solid Transportation Problem (MOSTP) with emission cost based on weight, budget, constraint and vehicle cost in crisp environment:

Here DM put a restriction on the transported amount $\mathrm{p}$ such that the DM consider those route, where the transported amount is greater than or equal to the restricted amount $\mathrm{p}$, otherwise DM cannot transport the amount through the route

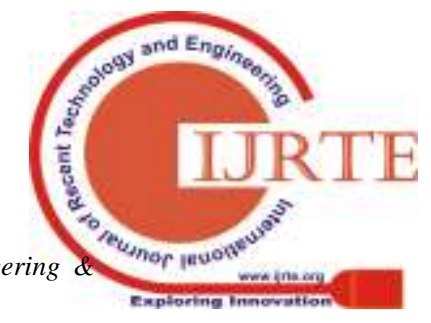




\section{MULTI-OBJECTIVE MULTI TIME SPAN FRACTIONAL CAPACITATED TRANSPORTATION PROBLEM IN THE TWO ECHELON SUPPLY CHAIN WITH MULTIFORM ITEMS AND MIXED CONSTRAINTS}

from the above concept. We formulate the following MOSTP

$$
\begin{aligned}
& \text { Min } \mathrm{f}_{1}=\sum_{\mathrm{I}=1}^{\mathrm{M}} \sum_{\mathrm{J}=1}^{\mathrm{N}} \sum_{\mathrm{K}=1}^{\mathrm{L}} e \mathrm{C}_{\mathrm{IJK}} e x_{\mathrm{IJK}} e \mathrm{z}_{\mathrm{iIJK}}+\sum_{\mathrm{I}=1}^{\mathrm{M}} \sum_{\mathrm{J}=1}^{\mathrm{N}} \sum_{\mathrm{K}=1}^{\mathrm{L}} \mathrm{V}\left(e x_{\mathrm{IJK}}\right) \\
& \text { Min } \mathrm{f}_{2}=\sum_{\mathrm{I}=1 \mathrm{~J}=1 \mathrm{~K}=1}^{\mathrm{N}} \sum_{\mathrm{K}=1}^{\mathrm{L}} e \mathrm{t}_{\mathrm{IJK}} e \mathrm{z}_{\mathrm{IJK}}, \operatorname{Min}_{3}=\sum_{\mathrm{I}=1 \mathrm{~J}=1 \mathrm{~K}=1}^{\mathrm{N}} \sum_{\mathrm{K}=1}^{\mathrm{L}} \mathrm{e}_{\mathrm{IJK}} e \mathrm{z}_{\mathrm{IJK}}
\end{aligned}
$$

Subject to constraints (2), (3), (4) and

$$
\sum_{\mathrm{I}=1}^{\mathrm{M}} \sum_{\mathrm{K}=1}^{\mathrm{L}} e \mathrm{C}_{\mathrm{IJK}} e x_{\mathrm{IJK}} e \mathrm{Z}_{\mathrm{IJK}} \leq e \mathrm{~B}_{\mathrm{J}}
$$

$e x_{\mathrm{IK}} \geq p, \forall \mathrm{I}, \mathrm{J}, \mathrm{K}$ where $p$ is any desired real value.”

\subsection{Model - III}

Multi-Objective Solid Transportation Problem (MOSTP) with Atanasov's triangular intuitionistic fuzzy number, emission cost based on weight, budget, constraint and vehicle cost:

In this model all supplies, demands conveyances capacities, unit transportation cost, time emission cost based on weight and budget at each customer as Atanasov's triangular intuitionistic fuzzy number. Here we formulate a MOSTP with M-origin, $\mathrm{N}$-destination and $\mathrm{K}$-conveyances as follows:

$$
\begin{aligned}
& \operatorname{Min} \mathrm{f}_{1}=\sum_{\mathrm{I}=1}^{\mathrm{M}} \sum_{\mathrm{J}=1}^{\mathrm{N}} \sum_{\mathrm{K}=1}^{\mathrm{L}} e \tilde{\mathrm{C}}_{\mathrm{IJK}} e x_{\mathrm{IJK}}+\sum_{\mathrm{I}=1}^{\mathrm{M}} \sum_{\mathrm{J}=1}^{\mathrm{N}} \sum_{\mathrm{K}=1}^{\mathrm{L}} \mathrm{V}\left(e x_{\mathrm{IJK}}\right) \\
& \operatorname{Min} \mathrm{f}_{2}=\sum_{\mathrm{I}=1 \mathrm{~J}=1}^{\mathrm{M}} \sum_{\mathrm{K}=1}^{\mathrm{N}} \sum_{\mathrm{e}}^{\mathrm{I}} \tilde{\mathrm{t}}_{\mathrm{JK}} e \mathrm{y}_{\mathrm{IJK}}, \operatorname{Min}_{3}=\sum_{\mathrm{I}=1}^{\mathrm{M}} \sum_{\mathrm{J}=1 \mathrm{~K}=1}^{\mathrm{N}} \sum_{\mathrm{IJK}}^{\mathrm{L}} \tilde{\mathrm{e}}_{\mathrm{IJ}} e y_{\mathrm{IJK}}
\end{aligned}
$$

Subject to constraints,

$$
\begin{aligned}
& \sum_{\mathrm{J}=1}^{\mathrm{N}} \sum_{\mathrm{K}=1}^{\mathrm{L}} e x_{\mathrm{IJK}} \leq e \tilde{\mathrm{a}}_{\mathrm{I}} \\
& \sum_{\mathrm{i}=1 \mathrm{k}=1}^{\mathrm{M}} \sum_{\mathrm{k}=1 \mathrm{~K} \mathrm{~K}}^{3} e \tilde{\mathrm{b}}_{\mathrm{j}} \\
& \sum_{\mathrm{i}=1 \mathrm{j}=1}^{\mathrm{M}} \sum_{\mathrm{j}=1}^{\mathrm{N}} e x_{\mathrm{ijk}} \leq e \tilde{\mathrm{e}}_{\mathrm{k}} \\
& \sum_{\mathrm{i}=1 \mathrm{k}=1}^{\mathrm{M}} \sum_{\mathrm{K}=1}^{\mathrm{K}} \mathrm{e} \tilde{\mathrm{C}}_{\mathrm{ijk}} \mathrm{e} x_{\mathrm{ijk}} \leq e \tilde{\mathrm{B}}_{\mathrm{j}} \\
& e \mathrm{x}_{\mathrm{ijk}} \geq 0, \forall \mathrm{i}, \mathrm{j}, \mathrm{k} .
\end{aligned}
$$

\subsection{Model - IV}

Restricted Multi-Objective Solid Transportation Problem (MOSTP) with Atanasov's triangular intuitionistic fuzzy number, emission cost, budget constraint and vehicle cost:

In this model all supplies, demands, conveyances capacities, unit transportation cost, time, emission cost based on weight and budget at each customer as Atanasov's triangular intuitionistic fuzzy number. Here we formulate a restricted MOSTP with M-origin, N-destination and $\mathrm{K}$-conveyances as follows:

$$
\operatorname{Min}_{1}=\sum_{\mathrm{i}=1}^{\mathrm{M}} \sum_{\mathrm{j}=1}^{\mathrm{N}} \sum_{\mathrm{k}=1}^{\mathrm{K}} e \tilde{\mathrm{C}}_{\mathrm{ijk}} e x_{\mathrm{ijk}} e \mathrm{Z}_{\mathrm{ijk}}+\sum_{\mathrm{i}=1}^{\mathrm{M}} \sum_{\mathrm{j}=1}^{\mathrm{N}} \sum_{\mathrm{k}=1}^{\mathrm{K}} \mathrm{V}\left(e x_{\mathrm{ijk}}\right)
$$

$$
\operatorname{Min} \mathrm{f}_{2}=\sum_{\mathrm{i}=1}^{\mathrm{M}} \sum_{\mathrm{j}=1}^{\mathrm{N}} \sum_{\mathrm{k}=1}^{\mathrm{K}} e \tilde{\mathrm{t}}_{\mathrm{ijk}} e \mathrm{z}_{\mathrm{ijk}}, \operatorname{Min} \mathrm{f}_{3}=\sum_{\mathrm{i}=1}^{\mathrm{M}} \sum_{\mathrm{j}=1}^{\mathrm{N}} \sum_{\mathrm{k}=1}^{\mathrm{K}} \tilde{\mathrm{e}}_{\mathrm{ijk}} e \mathrm{z}_{\mathrm{ijk}}
$$

Subject to constraints,

$$
\begin{aligned}
& \sum_{\mathrm{j}=1}^{\mathrm{N}} \sum_{\mathrm{k}=1}^{\mathrm{K}} e x_{\mathrm{ijk}} \leq e \tilde{\mathrm{a}}_{\mathrm{i}} \\
& \sum_{\mathrm{i}=1}^{\mathrm{M}} \sum_{\mathrm{k}=1}^{\mathrm{K}} e x_{\mathrm{ijk}}{ }^{3} e \tilde{\mathrm{b}}_{\mathrm{j}} \\
& \sum_{\mathrm{i}=1}^{\mathrm{M}} \sum_{\mathrm{j}=1}^{\mathrm{N}} e x_{\mathrm{ijk}} \leq e \tilde{\mathrm{e}}_{\mathrm{k}} \\
& \sum_{\mathrm{i}=1}^{\mathrm{M}} \sum_{\mathrm{k}=1}^{\mathrm{K}} e \tilde{\mathrm{C}}_{\mathrm{ijk}} e x_{\mathrm{ijk}} e \mathrm{z}_{\mathrm{ijk}} \leq e \tilde{\mathrm{B}}_{\mathrm{j}}
\end{aligned}
$$

(14)

$$
e \mathrm{x}_{\mathrm{ijk}}>\mathrm{p} \text {, where } \mathrm{p} \text { is any desired real value. }
$$

Using the methodology given in section 3 the above models are converted into single objective solid transportation problems and are solved using Lingo-13 Optimization Software.

\section{NUMERICAL EXAMPLE:}

A company produces a product at the two warehouses and this item is then shipped to two customers by three different modes of transport with different vehicle cost. The related transportation parameters of the proposed models are given below. Also for models $2 \& 4$ the transport amount is restricted to 10 units.

Table 1: Crisp Unit Transportation Cost and Time

\begin{tabular}{|l|l|l|l|l|l|l|}
\hline \multicolumn{7}{|l|}{ Unit Transportation Cost } \\
\hline \multirow{2}{*}{$\mathrm{i}$} & \multicolumn{2}{|l|}{$\mathrm{k}=1$} & $\mathrm{k}=2$ & \multicolumn{2}{l|}{$\mathrm{k}=3$} \\
\cline { 2 - 7 } & 1 & 2 & 1 & 2 & 1 & 2 \\
\hline 1 & 25 & 36 & 32 & 35 & 24 & 38 \\
\hline 2 & 34 & 36 & 36 & 35 & 25 & 31 \\
\hline & Unit Transportation Time & & \\
\hline 1 & 2 & 3 & 2 & 3 & 3 & 2 \\
\hline 2 & 2 & 3 & 2 & 3 & 2 & 2 \\
\hline
\end{tabular}

$e \mathrm{a}_{1}=60, e \mathrm{a}_{2}=90, e \mathrm{~b}_{1}=80, e \mathrm{~b}_{2}=70, e \mathrm{e}_{1}=50, e \mathrm{e}_{2}=$ $40, e \mathrm{e}_{3}=60, e \mathrm{~B}_{1}=3030$,

$e \mathrm{~B}_{2}=3040$.

$e \tilde{\mathrm{a}}_{1}=(59,60,63)(54,60,64), e \tilde{\mathrm{a}}_{2}=(89,90,91)(86$, 90, 94),

$e \tilde{\mathrm{b}}_{1}=(79,80,81)(76,80,84), e \tilde{\mathrm{b}}_{2}=(69,70,71)(66$, $70,74)$

$e \tilde{\mathrm{e}}_{1}=(49,50,52)(48$,

$50,53), e \tilde{\mathrm{e}}_{2}=(39,40,42)$

$(38,40,43)$ 
$e \tilde{\mathrm{e}}_{3}=(59,60,63)(54,60,64), e \tilde{\mathrm{B}}_{1}=(3023,3030$, 3037) $(3022,3030,3038)$

$e \tilde{\mathrm{B}}_{2}=(3033,3040,3047)(3032,3040,3048), e \tilde{\mathrm{C}}_{111}=$ $(20,25,30)(19,25,32)$

$e \tilde{\mathrm{C}}_{121}=(34,36,37)(32,36,38), e \tilde{\mathrm{C}}_{112}=(27,33,35)$ $(25,33,36)$

$e \tilde{\mathrm{C}}_{122}=(33,35,36)(31,35,37), e \tilde{\mathrm{C}}_{113}=(21,23,29)$

$(17,23,30)$

$e \tilde{\mathrm{C}}_{123}=(37,38,39)(35,38,40), e \tilde{\mathrm{C}}_{211}=(32,34,35)$

$(30,34,36)$

$e \tilde{\mathrm{C}}_{221}=(34,36,37)(32,36,38), e \tilde{\mathrm{C}}_{212}=(34,36,37)$ $(32,36,38)$

$e \tilde{\mathrm{C}}_{222}=(33,35,36)(31,35,37), e \tilde{\mathrm{C}}_{213}=(20,25,30)$

$(19,25,32)$

$e \tilde{\mathrm{C}}_{223}=(29,31,33)(30,31,34)$

$e \tilde{\mathrm{t}}_{111}=(.9,1.2,1.5)(.4,1.2,2), e \tilde{\mathrm{t}}_{121}=(1.7,2.35,3)(1.5$, $2.35,3.2)$

$e \tilde{\mathrm{t}}_{112}=(.6,1.3,2)(.4,1.3,2.2), \quad e \tilde{\mathrm{t}}_{122}=(1.7,2.35$,

3) $(1.5,2.35,3.2)$,

$e \tilde{\mathrm{t}}_{113}=(1.7,2.35,3)(1.5,2.35, .3 .2), e \tilde{\mathrm{t}}_{123}=(.9,1.2,1.5)$ $(.4,1.2,2)$,

$e \tilde{\mathrm{t}}_{211}=(.6,1.3,2)(.4,1.3,2.2), e \tilde{\mathrm{t}}_{221}=(1.7,2.35,3)$

$(1.5,2.35,3.2)$,

$e \tilde{\mathrm{t}}_{212}=(.7,1.45,2.2)(.5,1.45,2.4), \quad e \tilde{\mathrm{t}}_{222}=(1.7$,

$2.35,3)(1.5,2.35,3.2)$,

$e \tilde{\mathrm{t}}_{213}=(.7,1.35,2)(.5,1.35,2.2), e \tilde{\mathrm{t}}_{223}=(.9,1.2,1.5)$

$(.4,1.2,2), \mathrm{w}_{1}=0.5, \mathrm{w}_{2}=0.5$,

$\mathrm{v}=12$ and $\mathrm{v}_{\mathrm{c}}=6$.

\section{OPTIMAL RESULTS OF DIFFERENT MODELS:}

The optimal results for the different models with transporting amount are restricted to 10 units are as follows :

\begin{tabular}{|l|l|l|l|l|}
\hline & Model-I & Model-II & Model-III & Model-IV \\
\hline $\mathrm{f}_{1}$ & 4380 & 4874 & 4377 & 4848 \\
\hline $\mathrm{f}_{2}$ & 29 & 21 & 20.75 & 14.75 \\
\hline $\mathrm{f}_{3}$ & 0.24 & 0.18 & 0.19 & 0.135 \\
\hline$e \mathrm{x}_{111}$ & 50 & 10 & 50.25 & 10 \\
\hline$e \mathrm{x}_{211}$ & 0 & 10 & 0 & 10 \\
\hline$e \mathrm{x}_{121}$ & 0 & 10 & 0 & 10 \\
\hline$e \mathrm{x}_{221}$ & 0 & 20 & 0 & 19.75 \\
\hline$e \mathrm{x}_{112}$ & 0 & 10 & 0 & 10 \\
\hline$e \mathrm{x}_{212}$ & 0 & 10 & 0 & 10 \\
\hline$e \mathrm{x}_{122}$ & 0 & 10 & 0 & 10 \\
\hline$e \mathrm{x}_{222}$ & 40 & 10 & 39.75 & 10.25 \\
\hline$e \mathrm{x}_{113}$ & 10 & 10 & 9.75 & 10 \\
\hline$e \mathrm{x}_{213}$ & 20 & 30 & 20 & 30 \\
\hline$e \mathrm{x}_{123}$ & 0 & 10 & 0 & 10 \\
\hline$e \mathrm{x}_{223}$ & 30 & 10 & 30.25 & 10 \\
\hline & & & \multicolumn{2}{|l}{} \\
\hline
\end{tabular}

\section{ANALYSIS OF THE RESULTS}

In this paper, four mathematical models have been solved in which the emissions related to the transported quantity has been considered. Among these four models two (Model-I\& III) of them are without restriction on the transportation quantity and other two (Model-II\& IV) are with restriction on the transportation quantity. From the above table it has been notified that the total transportation cost increases and total transportation time as well as the total emission costs are decreased. If the Decision Maker (DM) imposed the restriction on transported amount, then the DM cannot transport the amount which is less than the limited quantity. For this reason, the transporting time of that particular type of route cannot be added into the total time. Due to this reason the total time is less than the total time of unrestricted models Similarly, the total emission cost based on weight is less than the total emission cost based on weight of unrestricted models, also that limited quantity can be accustomed throughout the routes where the amounts are transported and for this reason, the total cost is increased compared to the total cost of unrestricted models (i.e.,) Model-I and III.

\section{SENSITIVITY ANALYSIS}

As per expectation in real life problem, if $\mathrm{w} 1$ increases then the composite objective function value $\mathrm{Z}$ will increase. Also if w2 increases then the composite objective function $\mathrm{Z}$ will decrease. Similarly, w3 increases then the composite objective function $\mathrm{Z}$ will decrease. It is as per expectation in real life problem.

SENSITIVITY ANALYSIS OF MODEL-I

\begin{tabular}{|l|l|l|l|}
\hline $\mathrm{w}_{1}$ & $\mathrm{w}_{2}$ & $\mathrm{w}_{3}$ & $\mathrm{Z}=\mathrm{w}_{1} \mathrm{f}_{1}+\mathrm{w}_{2} \mathrm{f}_{2}+\mathrm{w}_{3} \mathrm{f}_{3}$ \\
\hline 0.1 & 0.5 & 0.4 & 452.6 \\
\hline 0.2 & 0.4 & 0.4 & 887.7 \\
\hline 0.3 & 0.4 & 0.3 & 1325.7 \\
\hline 0.4 & 0.3 & 0.3 & 1760.8 \\
\hline 0.5 & 0.3 & 0.2 & 2198.7 \\
\hline 0.6 & 0.2 & 0.2 & 2633.8 \\
\hline 0.7 & 0.2 & 0.1 & 3071.8 \\
\hline 0.8 & 0.1 & 0.1 & 3506.9 \\
\hline
\end{tabular}

\section{SENSITIVITY ANALYSIS OF MODEL-II}

\begin{tabular}{|l|l|l|l|}
\hline $\mathrm{w}_{1}$ & $\mathrm{w}_{2}$ & $\mathrm{w}_{3}$ & $\mathrm{Z}=\mathrm{w}_{1} \mathrm{f}_{1}+\mathrm{w}_{2} \mathrm{f}_{2}+\mathrm{w}_{3} \mathrm{f}_{3}$ \\
\hline 0.1 & 0.5 & 0.4 & 497.9 \\
\hline 0.2 & 0.4 & 0.4 & 983.3 \\
\hline 0.3 & 0.4 & 0.3 & 1470.7 \\
\hline 0.4 & 0.3 & 0.3 & 1955.9 \\
\hline 0.5 & 0.3 & 0.2 & 2443.3 \\
\hline 0.6 & 0.2 & 0.2 & 2928.6 \\
\hline 0.7 & 0.2 & 0.1 & 3416.0 \\
\hline 0.8 & 0.1 & 0.1 & 3901.3 \\
\hline
\end{tabular}

SENSITIVITY ANALYSIS OF MODEL-III 


\begin{tabular}{|l|l|l|l|}
\hline $\mathrm{w}_{1}$ & $\mathrm{w}_{2}$ & $\mathrm{w}_{3}$ & $\mathrm{Z}=\mathrm{w}_{1} \mathrm{f}_{1}+\mathrm{w}_{2} \mathrm{f}_{2}+\mathrm{w}_{3} \mathrm{f}_{3}$ \\
\hline 0.1 & 0.5 & 0.4 & 445.1 \\
\hline 0.2 & 0.4 & 0.4 & 883.8 \\
\hline
\end{tabular}

\begin{tabular}{|l|l|l|l|}
\hline 0.3 & 0.4 & 0.3 & 1321.5 \\
\hline 0.4 & 0.3 & 0.3 & 1757.1 \\
\hline 0.5 & 0.3 & 0.2 & 2194.8 \\
\hline 0.6 & 0.2 & 0.2 & 2630.4 \\
\hline 0.7 & 0.2 & 0.1 & 3068.1 \\
\hline 0.8 & 0.1 & 0.1 & 3503.7 \\
\hline
\end{tabular}

SENSITIVITY ANALYSIS OF MODEL-IV

\begin{tabular}{|l|l|l|l|}
\hline $\mathrm{w}_{1}$ & $\mathrm{w}_{2}$ & $\mathrm{w}_{3}$ & $\mathrm{Z}=\mathrm{w}_{1} \mathrm{f}_{1}+\mathrm{w}_{2} \mathrm{f}_{2}+\mathrm{w}_{3} \mathrm{f}_{3}$ \\
\hline 0.1 & 0.5 & 0.4 & 492.2 \\
\hline 0.2 & 0.4 & 0.4 & 975.6 \\
\hline 0.3 & 0.4 & 0.3 & 1460.3 \\
\hline 0.4 & 0.3 & 0.3 & 1943.7 \\
\hline 0.5 & 0.3 & 0.2 & 2428.5 \\
\hline 0.6 & 0.2 & 0.2 & 2911.8 \\
\hline 0.7 & 0.2 & 0.1 & 3396.6 \\
\hline 0.8 & 0.1 & 0.1 & 3879.9 \\
\hline
\end{tabular}

\section{CONCLUSION}

The most important intend of this paper is to present the emission cost based on weight of the quantity transported and the solution procedure related to with and without restriction on the transported quantity in the multi-objective solid transportation problem. Here the unit transportation penalties, demand, source, capacity budget at each destination and emission cost based on weight are measured as triangular Atanasov's Intuitionistic Fuzzy Number. The average system is used to convert the models III and IV into crisp equivalent model. In numerical example, all the models are resolved using LINGO 13.0 Optimization Software. From the results, it has been observed that, the optimal value of the fuzzy model is $1.42 \%$ lesser than the crisp model so, the fuzzy models are better than the crisp models. Also from the results of the models II \&IV it is observed that the emission cost is less when the restriction on the transportation quantity is imposed. These models are highly supportive to the society in reducing the co 2 emissions and preserve the nature.

\section{REFERENCES}

1. Abhijit Baidya, UttamkumarBera, ManoranjanMaiti,Uncertain multi-objective restricted solid transportation problem with budget and vehicle cost ,U.P.B. sci.Bull.,series c,vol.78,ISS.1,2016.

2. A.Gupta and A. Kumar, A new method for solving linear multi-objective transportation problems with fuzzy parameters, Applied Mathematical Modelling36 (2012), 1421-1430.

3. A.Nagoorgani and R. Ponnalagu, A new approach on solving intuitionistic fuzzy linear programming problem, Applied Mathematical Sciences 6 (2012), $3467-3474$.

4. A.I. Ban, Intuitionistic fuzzy-valued possibility and necessity measures, Eighth International Conference on IFSs, Varna 10 (2004), 1-7.

5. A.N. Gani and S. Abbas, Solving intuitionstic fuzzy transportation problem using zero suffix algorithm, International Journal of Mathematics Sciences \&EnggineeringApplications6(2012), 73-82.

6. Bit, A.K., Biswal, M.P. and Alam, S.S. "Fuzzy programming approach to multi-objective solid transportation problem", Fuzzy Sets and Systems, vol. 57, pp. 183-194(1993).

7. Christophe Rizet, Cecila Cruz, Maiame Mbacke,Reducing Freight Transport co2 emission by increasing the load factor, Procedia-Social science and behavioral Science48(2012) 184-195

8. Dipankarchakraborty,Dipakkumar Jana and Tapan Kumar Roy,A new approach to solve multi-objective multi-choice multi-item atanassov's intuitionistic fuzzy transportation problem using chance operator ,journal of intelligent \& fuzzy systems 28(2015)843-865.

9. D. Dubey and A. Mehra, Linear programming with triangular intuitionistic fuzzy number, Advances in Intelligent SystemsResearch1 (2011), 563-569.

10. Elmaghraby S.E., Allocation under uncertainty when the demand has continuous distribution function, Management Science, vol. 6, pp. 270-294(1960).

11. G.S. Mahapatra and T.K. Roy, Intuitionistic fuzzy number and its arithmetic operation with application on system failure, Journal of Uncertain Systems 7 (2013), 92-107.

12. Haley, K.B., "The multi-index problem", Operations Research,vol. 11,pp. 368-379(1963).

13. Haley, K.B.,"The solid transportation problem”, Operations Research,vol. 10, pp. 448-463(1962).

14. Hitchcock.F.L,The distribution of a product from several sources to numerous localities, Journal Mathematical Physics20 (1941), 224-230

15. LiY.,Ida K., Gen M. and Kobuchi, R.,"Improved genetic algorithm for solving multi objective solid transportation problem with fuzzy numbers", Computers and Industrial Engineering,vol. 33, pp. 589-592(1997).

16. R.J. Hussain and S.P. Kumar, Algorithmic approach for solving intuitionistic fuzzy transportation problem, Applied Mathematical Sciences 6 (2012), 3981-3989.

17. Schell, E.,"Distribution of a product by several properties, in: Directorate of Management Analysis", Proceedings of the Second Symposium in Linear Programming", vol. 2, pp.615-642, DCS / Comptroller H.Q.U.S.A.F.

18. Zadeh, L.A., "Fuzzy sets as a basis for a theory of possibility", Fuzzy Sets and Systems, vol. 1, pp. 3- 28(1978).

19. Zimmermann, H.-J., "Fuzzy programming and linear programming with several objective functions", Fuzzy Sets and Systems, vol. 1, pp. 45-55 (1978). 